\title{
Radiation from an accelerated quark via AdS/CFT
}

\author{
Kengo Maeda, , and Takashi Okamura, 田 \\ ${ }^{1}$ Department of Engineering, Shibaura Institute of Technology, Saitama, 330-8570, Japan \\ ${ }^{2}$ Department of Physics, Kwansei Gakuin University, Sanda, 669-1337, Japan
}

(Dated: November 1, 2018)

\begin{abstract}
In this paper we investigate radiation by an accelerated quark in a strongly coupled gauge theory via AdS/CFT correspondence. According to AdS/CFT dictionary, we can read off energy density or energy flux of the radiation from asymptotic gravitational field in AdS bulk sourced by an accelerated string trailing behind the quark. In the case of an oscillating quark with frequency $\Omega$, we show that the time averaged energy density is asymptotically isotropic and it falls off as $\left(g_{\mathrm{YM}}^{2} N\right)^{1 / 2} \Omega^{4} / R^{2}$ with distance $R$ from the source. In a toy model of a scattered quark by an external field, we simply estimate Poynting vector by the bremsstrahlung radiation and show that the energy flux is anisotropic outgoing radiation. Based on these investigations, we discuss the properties of strongly coupled gauge theory radiation in comparison with electromagnetic radiation.
\end{abstract}

PACS numbers: 11.25.Tq, 04.20.-q

\section{INTRODUCTION}

It is quite interesting to investigate the mechanism of energy dissipation by an external quark moving through gauge theory plasma from both theoretical and experimental point of view. Recently, a strongly coupled gauge theory plasma is believed to be formed at RHIC [1, 2]. A heavy quark moving through the plasma is considered to lose the energy via two-body collisions with thermal quarks or gluon emission. Although it is detectable through jet-quenching effect at RHIC, this energy loss rate cannot be calculated analytically beyond the perturbative QCD.

Gauge/gravity duality [3, 4, 5] shed some light on the investigation of the strongly coupled gauge theories. This duality states that the expectation value of the energy-momentum tensor $\left\langle T_{\mu \nu}\right\rangle$ of thermal $\mathcal{N}=4$ super-Yang-Mills (SYM) theory at large 't Hooft coupling $g_{\mathrm{YM}}^{2} N$ and a large number of colors $N$ can be read off from the perturbed metric of anti-de Sitter (AdS) black hole. For example, we can obtain transport coefficients such as shear viscosity by calculating quasi-normal mode of AdS black hole. Many calculations showed that the ratio of viscosity divided by entropy density is equal to $1 / 4 \pi$ for a large class of gauge theories described by gravity duals (See, for example, Ref. [6, 7] for all references). This universal value is in good agreement with the RHIC data, which suggests that it is a universal feature for all strongly coupled gauge theories, including QCD. This agreement leads us to seek other universal properties of the strongly coupled plasmas. Even though there is no universality, we would obtain some insights which are not readily obtained from perturbative techniques and lattice simulations.

In gauge/gravity duality, a heavy quark corresponds to an open string which stretches from the boundary of AdS spacetime to the horizon of the black hole. Under the test string approximation, a large number of studies have been made on estimating a jet-quenching parameter or energy loss rate of the quark moving through the plasma via gauge/gravity duality $8,8,9,10,11,12,13,114,15]$. Beyond the test string approximation, we need to take into account the back-reaction by the radiation from the string onto the original AdS geometry, as pointed out in [15]. So, a good place to start is to

*Electronic address: maeda302@sic.shibaura-it.ac.jp

$\dagger$ Electronic address: tokamura@kwansei.ac.jp 
investigate the properties of radiation emitted by the string. In a series of papers [16, 17, 18, 19, 20], the energy density and energy flux associated with the quark with constant velocity were calculated from the metric perturbation by the string trailing behind the quark. In the case of supersonic motion, the Mach cone structure, which is characteristic of Cherenkov radiation, was also displayed.

In this paper we shall investigate the properties of radiation from an accelerated quark via gauge/gravity duality. Since the quarks in the plasma would be scattered by one another, the bremsstrahlung radiation by the scattered quark would bring about another mechanism of energy dissipation of the quark moving through the plasma. Callan and Güijosa 21] first investigated timedependent fields produced by an oscillating quark via gauge/gravity duality and showed that the one point function $\left\langle\operatorname{Tr} F^{2}\right\rangle$ in the boundary theory decays faster than $1 / R^{2}$. This does not immediately mean that there is no radiation emitted by the quark. As mentioned in [21, 22], we can explain the reason for the curious behavior of the function by analogy with electromagnetism as follows: $F^{2}$ is expressed by the electric field $E$ and magnetic field $B$ as $F^{2}=E^{2}-B^{2}$. Since $E^{2}=B^{2} \sim 1 / R^{2}$ for radiation field, the leading radiation parts are cancelled in $F^{2}$. So, we show the existence of the radiation by dealing with energy-momentum tensor of the gauge theory generated by an accelerated quark.

As a first step, we calculate energy density or energy flux of radiation from an accelerated quark at zero temperature by solving master equations of linearized Einstein equations sourced by the string given in [19]. In section [1 we briefly review the master equation and the general method to solve the equation developed in [19]. In section [II] we calculate the time averaged energy density $\left\langle T_{00}\right\rangle$ of radiation in the case of an oscillating quark with frequency $\Omega$. It is shown that the energy density is asymptotically isotropic and it falls off as $\sqrt{g_{\mathrm{YM}}^{2} N} \Omega^{4} / R^{2}$. In section IV we simply estimate the Poynting vector associated with the bremsstrahlung radiation in a toy model of a scattered quark by an external field. It is shown that the energy flux is anisotropic outgoing radiation. Conclusion and discussions are devoted in the final section.

\section{MASTER EQUATION FOR A SCALAR MODE}

A quark in $\mathcal{N}=4 \mathrm{SYM}$ theory at zero temperature corresponds to a fundamental string through the Nambu-Goto action

$$
S=-\frac{1}{2 \pi \alpha^{\prime}} \int d \sigma^{2} \sqrt{-h}
$$

where $h$ is the determinant of the world-sheet metric $h_{\alpha \beta}=g_{\mu \nu} \partial_{\alpha} X^{\mu} \partial_{\beta} X^{\nu}$. The background metric $g$ is given by the metric of $A d S_{5}$ spacetime

$$
d s^{2}=a(r)^{2}\left(-d t^{2}+d x_{i}^{2}\right)+d r^{2} / a(r)^{2}, \quad a(r)=r / L, \quad(i=1,2,3),
$$

where $L$ and $\alpha^{\prime}$ are related by 't Hooft coupling $g_{\mathrm{YM}}^{2} N$ as

$$
L^{4} / \alpha^{\prime 2}=g_{\mathrm{YM}}^{2} N .
$$

We solve the linearized Einstein equations in the metric sourced by the accelerated string. Since we are interested in the energy density or energy flux of the bremsstrahlung radiation, we shall focus on the scalar perturbations of the linearized Einstein equations. Following [19], the scalar modes of 
the string energy-momentum tensor $\tau_{\mu \nu}$ and the metric perturbations $h_{\mu \nu}$ are expressed as

$$
\begin{aligned}
& \tau_{a b}(t, \boldsymbol{x}, r)=\int d \omega \int \frac{d^{3} k}{(2 \pi)^{3}} \tau_{b c}^{S}(\omega, \boldsymbol{k}, r) \mathbb{S}(\boldsymbol{k}, \boldsymbol{x}) e^{-i \omega t} \\
& \tau_{b i}(t, \boldsymbol{x}, r)=a(r) \int d \omega \int \frac{d^{3} k}{(2 \pi)^{3}}\left[\tau_{b}^{S}(\omega, \boldsymbol{k}, r) \mathbb{S}_{i}(\boldsymbol{k}, \boldsymbol{x})\right] e^{-i \omega t} \\
& \tau_{i j}(t, \boldsymbol{x}, r)=2 a^{2}(r) \int d \omega \int \frac{d^{3} k}{(2 \pi)^{3}}\left[p^{S}(\omega, \boldsymbol{k}, r) \delta_{i j} \mathbb{S}(\boldsymbol{k}, \boldsymbol{x})+\tau^{S}(\omega, \boldsymbol{k}, r) \mathbb{S}_{i j}(\boldsymbol{k}, \boldsymbol{x})\right] e^{-i \omega t}
\end{aligned}
$$

and

$$
\begin{aligned}
& h_{a b}(t, \boldsymbol{x}, r)=\int d \omega \int \frac{d^{3} k}{(2 \pi)^{3}} f_{a b}^{S}(\omega, \boldsymbol{k}, r) \mathbb{S}(\boldsymbol{k}, \boldsymbol{x}) e^{-i \omega t}, \\
& h_{b i}(t, \boldsymbol{x}, r)=a(r) \int d \omega \int \frac{d^{3} k}{(2 \pi)^{3}}\left[f_{b}^{S}(\omega, \boldsymbol{k}, r) \mathbb{S}_{i}(\boldsymbol{k}, \boldsymbol{x})\right] e^{-i \omega t}, \\
& h_{i j}(t, \boldsymbol{x}, r)=2 a^{2}(r) \int d \omega \int \frac{d^{3} k}{(2 \pi)^{3}}\left[H_{L}^{S}(\omega, \boldsymbol{k}, r) \delta_{i j} \mathbb{S}(\boldsymbol{k}, \boldsymbol{x})+H_{T}^{S}(\omega, \boldsymbol{k}, r) \mathbb{S}_{i j}(\boldsymbol{k}, \boldsymbol{x})\right] e^{-i \omega t},
\end{aligned}
$$

where $a, b=t, r$ and functions $\mathbb{S}, \mathbb{S}_{i}$, and $\mathbb{S}_{i j}$ are defined as

$$
\begin{array}{ll}
\mathbb{S}(\boldsymbol{k}, \boldsymbol{x})=e^{i \boldsymbol{k} \cdot \boldsymbol{x}}, & \mathbb{S}_{i}(\boldsymbol{k}, \boldsymbol{x})=-\frac{1}{k} \partial_{i} \mathbb{S}(\boldsymbol{k}, \boldsymbol{x}), \\
\mathbb{S}_{i j}(\boldsymbol{k}, \boldsymbol{x})=\frac{1}{k^{2}} \partial_{i} \partial_{j} \mathbb{S}(\boldsymbol{k}, \boldsymbol{x})+\frac{1}{3} \delta_{i j} \mathbb{S}(\boldsymbol{k}, \boldsymbol{x}) . &
\end{array}
$$

Here $k$ is defined by $k:=|\boldsymbol{k}|$.

The diffeomorphism-invariant quantity of $f_{a b}^{S}$ is constructed as [19]

$$
\hat{f}_{a b}^{S}=f_{a b}^{S}+2 D_{(a} X_{b)}, \quad X_{a}=\frac{a(r)}{k}\left(f_{a}^{S}+\frac{a(r)}{k} \partial_{a} H_{T}^{S}\right) .
$$

Defining the master field ${ }^{1}$ as

$$
\Phi(\omega, \boldsymbol{k}, r)=-\frac{k^{2}}{2 a^{3} L}\left(\frac{\hat{f}_{t t}^{S}}{a}-a^{3} \hat{f}_{r r}^{S}+\frac{a^{3} \hat{f}_{t r}^{S}}{i r \omega}\right),
$$

the master equation is given by

$$
\mathcal{L} \Phi(\omega, \boldsymbol{k}, r):=\left(\partial_{r}^{2}+\frac{5}{r} \partial_{r}-\frac{L^{4}}{r^{4}}\left(k^{2}-\omega^{2}\right)+\frac{4}{r^{2}}\right) \Phi(\omega, \boldsymbol{k}, r)=-j(\omega, \boldsymbol{k}, r),
$$

with a source term of the string

$$
\begin{aligned}
j(\omega, \boldsymbol{k}, r)=\frac{1}{3 L^{3} \omega r^{3}} & {\left[k^{2} L^{2} \omega r^{3} \tau_{r r}(\omega, \boldsymbol{k}, r)+2 \omega\left(3 L^{4} \omega^{2} r-4 k^{2} L^{4} r+48 r^{3}\right) \tau^{S}(\omega, \boldsymbol{k}, r)\right.} \\
& \left.-i k L\left\{-3 i \omega r^{3} \tau_{r}^{S}(\omega, \boldsymbol{k}, r)+\left(k^{2} L^{4}-9 r^{2}\right) \tau_{t}^{S}(\omega, \boldsymbol{k}, r)\right\}\right] \\
-\frac{i}{3 L^{3} \omega r} & {\left[2 k^{2} L^{2} \tau_{t r}^{S}(\omega, \boldsymbol{k}, r)+k L r\left\{k L \tau_{t r}^{\prime S}(\omega, \boldsymbol{k}, r)-3 \tau_{t}^{\prime S}(\omega, \boldsymbol{k}, r)\right\}\right.} \\
+ & \left.6 i r^{2} \omega\left\{9 \tau^{\prime S}(\omega, \boldsymbol{k}, r)+r \tau^{\prime \prime S}(\omega, \boldsymbol{k}, r)\right\}\right]
\end{aligned}
$$

\footnotetext{
${ }^{1}$ We set $\kappa_{5}^{2}=1$
} 
where a prime means the derivative with respect to $r$.

Eq. (II.13) can be solved by a Green function $G_{R}\left(\omega, \boldsymbol{k}, r ; r_{0}\right)$ which obeys

$$
\mathcal{L} G_{R}\left(\omega, \boldsymbol{k}, r ; r_{0}\right)=\delta\left(r-r_{0}\right) .
$$

This is constructed by two solutions of the homogeneous equation $\mathcal{L} \Phi_{H}=0=\mathcal{L} \Phi_{B}$, where $\Phi_{H}$ satisfies appropriate boundary conditions at the degenerate horizon $r=0$, while $\Phi_{B}$ has appropriate falloff at the boundary of $\mathrm{AdS}_{5}$. Then the Green function $G_{R}$ satisfying the boundary conditions at both the horizon and infinity is represented by $\Phi_{B}$ and $\Phi_{H}$ as

$$
G_{R}\left(\omega, \boldsymbol{k}, r ; r_{0}\right)=\frac{1}{W\left[\Phi_{H}, \Phi_{B}\right]\left(r_{0}\right)}\left[\Phi_{H}\left(r_{0}\right) \Phi_{B}(r) \theta\left(r-r_{0}\right)+\Phi_{H}(r) \Phi_{B}\left(r_{0}\right) \theta\left(r_{0}-r\right)\right],
$$

where $W\left[\Phi_{H}, \Phi_{B}\right]\left(r_{0}\right)$ is the Wronskian defined by

$$
W\left[\Phi_{H}, \Phi_{B}\right](r)=\Phi_{H}(r) \partial_{r} \Phi_{B}(r)-\Phi_{B}(r) \partial_{r} \Phi_{H}(r) .
$$

So, the solution of Eq. (II.13) is given by

$$
\Phi(\omega, \boldsymbol{k}, r)=A(r) \Phi_{H}(r)+B(r) \Phi_{B}(r)
$$

with

$$
A(r)=-\int_{r}^{\infty} d r_{0} \frac{\Phi_{B}\left(r_{0}\right)}{W\left(r_{0}\right)} j\left(r_{0}\right), \quad B(r)=-\int_{0}^{r} d r_{0} \frac{\Phi_{H}\left(r_{0}\right)}{W\left(r_{0}\right)} j\left(r_{0}\right) .
$$

As discussed in [20], the expectation value $\left\langle T_{\mu \nu}(t, \boldsymbol{x})\right\rangle$ of the energy-momentum tensor of $\mathcal{N}=4$ SYM theory can be read off from the asymptotic behavior of $\Phi(\omega, \boldsymbol{k}, r)$ near $\mathrm{AdS}_{5}$ boundary,

$$
\Phi(\omega, \boldsymbol{k}, r)=\frac{P(\omega, \boldsymbol{k})}{r}+\frac{Q(\omega, \boldsymbol{k})}{r^{2}}+O\left(r^{-3}\right) .
$$

The term $P / r$ in Eq. (II.20) produces singularity of $\left\langle T_{\mu \nu}(t, \boldsymbol{x})\right\rangle$ supported at the location of the quark, while the $Q / r^{2}$ term gives the energy density of the radiation as

$$
\left\langle T_{00}(\omega, \boldsymbol{k})\right\rangle=Q(\omega, \boldsymbol{k}) .
$$

\section{RADIATION FROM AN OSCILLATING STRING}

In this section, we calculate the energy density $\left\langle T_{00}\right\rangle$ of $\mathcal{N}=4 \mathrm{SYM}$ radiation from an oscillating quark. This can be obtained by solving Eq. (II.13) for an oscillating open string.

Now we consider that the string oscillates only in the $x_{1}$ direction $\left(X_{2}=X_{3}=0\right)$. Under the static gauge $\tau=t, \quad \sigma=z=L^{2} / r$, the equation of motion is obtained from Eq. (II.1) as

$$
z^{2} \frac{\partial}{\partial z}\left(\frac{X_{1}^{\prime}}{z^{2} \sqrt{1+X_{1}^{\prime 2}-\dot{X}_{1}^{2}}}\right)-\frac{\partial}{\partial t}\left(\frac{z^{2} \dot{X}_{1}}{\sqrt{1+X_{1}^{\prime 2}-\dot{X}_{1}^{2}}}\right)=0 .
$$

Here a dot and a prime mean the derivatives with respect to $t$ and $z$, respectively. Assuming that the oscillations are very small $\left(\left|X_{1}\right| \ll 1\right)$, the linearized equation is simply given by

$$
X_{1}^{\prime \prime}-\frac{2}{z} X_{1}^{\prime}-\ddot{X}_{1}=0 .
$$


Under the ansatz $X_{1}(t, z)=\sin (\Omega t) \xi(z)$, it is easily checked that

$$
\xi(z)=\epsilon\{\cos (\Omega z)+\Omega z \sin (\Omega z)\}, \quad z=L^{2} / r,
$$

is a solution of Eq. (III.2), where $\epsilon$ is a small parameter and $\Omega$ is a positive constant, representing the angular frequency of the oscillating string. Calculating the energy-momentum tensor $\tau^{\mu \nu}$,

$$
\tau^{\mu \nu}=\frac{2}{\sqrt{-g}} \frac{\delta S}{\delta g_{\mu \nu}}=-\frac{1}{2 \pi \alpha^{\prime}} \frac{\sqrt{-h}}{\sqrt{-g}} h^{\alpha \beta}\left(\partial_{\alpha} X^{\mu}\right)\left(\partial_{\beta} X^{\nu}\right) \delta\left(x^{1}-X^{1}\right) \delta\left(x^{2}\right) \delta\left(x^{3}\right),
$$

one gets the non-zero components up to the second order

$$
\begin{aligned}
& \tau^{t t}=\frac{1}{4 \pi \alpha^{\prime} a^{5}(r)} \int \frac{d^{3} k}{(2 \pi)^{3}} e^{i \boldsymbol{k} \cdot \boldsymbol{x}}\left(2-2 i k_{1} \sin (\Omega t) \xi(r)+\Omega^{2} \cos ^{2}(\Omega t) \xi^{2}(r)\right. \\
& \left.+a^{4}(r) \sin ^{2}(\Omega t) \xi^{\prime 2}(r)-k_{1}^{2} \sin ^{2}(\Omega t) \xi^{2}(r)\right), \\
& \tau^{r r}=\frac{-1}{4 \pi \alpha^{\prime} a(r)} \int \frac{d^{3} k}{(2 \pi)^{3}} e^{i \boldsymbol{k} \cdot \boldsymbol{x}}\left(2+2 i k_{1} \sin (\Omega t) \xi(r)-\Omega^{2} \cos ^{2}(\Omega t) \xi^{2}(r)\right. \\
& \left.-a^{4}(r) \sin ^{2}(\Omega t) \xi^{\prime 2}(r)-k_{1}^{2} \sin ^{2}(\Omega t) \xi^{2}(r)\right), \\
& \tau^{r t}=-\frac{\Omega}{2 \pi \alpha^{\prime} a(r)} \cos (\Omega t) \sin (\Omega t) \xi(r) \xi^{\prime}(r) \int \frac{d^{3} k}{(2 \pi)^{3}} e^{i \boldsymbol{k} \cdot \boldsymbol{x}}, \\
& \tau^{t 1}=\frac{\Omega}{2 \pi \alpha^{\prime} a^{5}(r)} \cos (\Omega t) \xi(r)\left(1-i k_{1} \sin (\Omega t) \xi(r)\right) \int \frac{d^{3} k}{(2 \pi)^{3}} e^{i \boldsymbol{k} \cdot \boldsymbol{x}}, \\
& \tau^{r 1}=-\frac{1}{2 \pi \alpha^{\prime} a(r)} \sin (\Omega t) \xi^{\prime}(r)\left(1-i k_{1} \sin (\Omega t) \xi(r)\right) \int \frac{d^{3} k}{(2 \pi)^{3}} e^{i \boldsymbol{k} \cdot \boldsymbol{x}}, \\
& \tau^{11}=\frac{1}{2 \pi \alpha^{\prime} a^{5}(r)}\left(\Omega^{2} \cos ^{2}(\Omega t) \xi^{2}(r)-a^{4}(r) \sin ^{2}(\Omega t) \xi^{\prime}(r)^{2}\right) \int \frac{d^{3} k}{(2 \pi)^{3}} e^{i \boldsymbol{k} \cdot \boldsymbol{x}},
\end{aligned}
$$

where a dash denotes the derivative with respect to $r$. Substituting these into Eqs. (II.4)-(II.6), the components $\tau^{S}, P^{S}, \tau_{t}^{S}, \tau_{r}^{S}, \tau_{r r}^{S}$, and $\tau_{t r}^{S}$ are

$$
\begin{aligned}
\tau_{t}^{S} & =-\frac{i \Omega k_{1} \xi}{4 \pi \alpha^{\prime} k a^{2}(r)}\left[\delta(\omega+\Omega)+\delta(\omega-\Omega)-k_{1} \xi \frac{\delta(\omega+2 \Omega)-\delta(\omega-2 \Omega)}{2}\right] \\
\tau_{r}^{S} & =-\frac{k_{1} \xi^{\prime}}{4 \pi \alpha^{\prime} k a^{2}(r)}\left[\delta(\omega+\Omega)-\delta(\omega-\Omega)+k_{1} \xi \delta(\omega)-k_{1} \xi \frac{\delta(\omega+2 \Omega)+\delta(\omega-2 \Omega)}{2}\right] \\
P^{S} & =\frac{1}{24 \pi \alpha^{\prime} a(r)}\left[\left(\frac{\Omega^{2}}{a^{2}(r)} \xi^{2}-a^{2}(r) \xi^{\prime 2}\right) \delta(\omega)+\left(\frac{\Omega^{2}}{a^{2}(r)} \xi^{2}+a^{2}(r) \xi^{\prime 2}\right) \frac{\delta(\omega+2 \Omega)+\delta(\omega-2 \Omega)}{2}\right] \\
\tau^{S} & =-\frac{3}{2}\left(\frac{3 k_{1}^{2}}{k^{2}}-1\right) P^{S}, \\
\tau_{r r}^{S} & =\frac{1}{8 \pi \alpha^{\prime} a^{5}(r)}\left[\left(a^{4}(r) \xi^{\prime 2}+k_{1}^{2} \xi^{2}+\Omega^{2} \xi^{2}-4\right) \delta(\omega)+k_{1} \xi \frac{\delta(\omega+\Omega)-\delta(\omega-\Omega)}{2}\right. \\
\quad & \left.+\left(\Omega^{2} \xi^{2}-a^{4}(r) \xi^{\prime 2}-k_{1}^{2} \xi^{2}\right) \frac{\delta(\omega+2 \Omega)+\delta(\omega-2 \Omega)}{2}\right] \\
\tau_{t r}^{S} & =-\frac{i \Omega}{8 \pi \alpha^{\prime} a(r)} \xi \xi^{\prime}(\delta(\omega+2 \Omega)-\delta(\omega-2 \Omega)) .
\end{aligned}
$$


We are interested in the time integral of the energy density,

$$
\int_{-\infty}^{\infty} d t\left\langle T_{00}(t, \boldsymbol{x})\right\rangle=\int_{-\infty}^{\infty} d t \int_{-\infty}^{\infty} d \omega\left\langle T_{00}(\omega, \boldsymbol{x})\right\rangle e^{-i \omega t}=2 \pi\left\langle T_{00}(\omega=0, \boldsymbol{x})\right\rangle .
$$

Owing to the linearity of Eq.(II.13), only the terms with $\delta(\omega)$ in Eq.(III.6) contribute to the time integral of the energy density. So, we drop the terms with $\delta(\omega \pm \Omega)$ or $\delta(\omega \pm 2 \Omega)$ in Eq.(III.6). Substituting Eq. (III.3) and Eq. (III.6) into the source term (II.14), one obtains

$$
\begin{aligned}
j(\omega, \boldsymbol{k}, r) & =j_{0}(\boldsymbol{k}, r) \delta(\omega)+\cdots, \\
j_{0}(\boldsymbol{k}, r) & =\epsilon^{2}\left(\frac{C_{1}}{r^{3}}+\frac{C_{2}}{r^{5}}+\frac{C_{3}}{r^{7}}\right) \cos (2 \Omega z) \\
& +\epsilon^{2}\left(\frac{S_{1}}{r^{4}}+\frac{S_{2}}{r^{6}}\right) \sin (2 \Omega z) \\
& +\epsilon^{2}\left(\frac{N_{1}}{r^{3}}+\frac{N_{2}}{r^{5}}+\frac{N_{3}}{r^{7}}\right),
\end{aligned}
$$

where all the coefficients are given by

$$
\begin{aligned}
C_{1} & =\frac{\Omega^{2}}{16 \pi \alpha^{\prime}}\left(1-\frac{3 k_{1}^{2}}{k^{2}}\right), \quad C_{2}=\frac{L^{4}}{48 \pi \alpha^{\prime}}\left(18 \Omega^{4} \frac{k_{1}^{2}}{k^{2}}+6 \Omega^{2}\left(k_{1}^{2}-\Omega^{2}\right)+k^{2}\left(k_{1}^{2}-3 \Omega^{2}\right)\right), \\
C_{3} & =\frac{L^{8} \Omega^{2}}{48 \pi \alpha^{\prime}}\left[-k^{2} k_{1}^{2}+8 \Omega^{2}\left(k^{2}+3 \Omega^{2}\right)\left(1-\frac{3 k_{1}^{2}}{k^{2}}\right)\right], \\
S_{1} & =\frac{L^{2} \Omega^{3}}{8 \pi \alpha^{\prime}}\left(1-\frac{3 k_{1}^{2}}{k^{2}}\right), \quad S_{2}=\frac{L^{6} \Omega}{24 \pi \alpha^{\prime}}\left(k^{2} k_{1}^{2}-3 \Omega^{2}\left(k^{2}-3 k_{1}^{2}\right)+6 \frac{\Omega^{4}}{k^{2}}\left(k^{2}-3 k_{1}^{2}\right)\right), \\
N_{1}=\frac{\Omega^{2}}{16 \pi \alpha^{\prime}}\left(1-\frac{3 k_{1}^{2}}{k^{2}}\right), & N_{2}=\frac{L^{4} k^{2}}{48 \pi \alpha^{\prime}}\left(6 \Omega^{2} \frac{k_{1}^{2}}{k^{2}}+k_{1}^{2}-3 \Omega^{2}\right), \\
N_{3}=\frac{L^{8} \Omega^{2}}{48 \pi \alpha^{\prime}} k^{2}\left(k_{1}^{2}+2 \Omega^{2}\right) . &
\end{aligned}
$$

The two-independent homogeneous solutions $\Phi_{H}$ and $\Phi_{B}$ for $\omega=0$ are simply represented by the modified Bessel function as

$$
\Phi_{B}(r)=\frac{I_{0}\left(k L^{2} / r\right)}{r^{2}}, \quad \Phi_{H}(r)=\frac{K_{0}\left(k L^{2} / r\right)}{r^{2}},
$$

and their Wronskian is simply given by

$$
W=-1 / r^{5} .
$$

We can obtain the coefficient $Q$ in Eq. (II.20) through a straightforward but tedious calculation (the detailed calculation is given in the Appendix A). The coefficient $Q$ is given by

$$
\begin{aligned}
Q(\omega, \boldsymbol{k}) & =Q_{0}(\boldsymbol{k}) \delta(\omega)+\cdots, \\
Q_{0}(\boldsymbol{k}) & =\frac{\pi \epsilon^{2}}{2 L^{2} \Omega} \frac{C_{2}}{\left(4+k^{2} / \Omega^{2}\right)^{1 / 2}}+\frac{3 \pi \epsilon^{2}}{2 L^{6} \Omega^{5}} \frac{C_{3} k^{2}}{\left(4+k^{2} / \Omega^{2}\right)^{5 / 2}}-\frac{\pi \epsilon^{2}}{L^{6} \Omega^{3}} \frac{C_{3}}{\left(4+k^{2} / \Omega^{2}\right)^{3 / 2}} \\
& +\frac{\pi \epsilon^{2}}{2} S_{1} \ln \left(\frac{2 \Omega}{k}+\sqrt{\frac{4 \Omega^{2}}{k^{2}}+1}\right)+\frac{\pi \epsilon^{2}}{L^{4}} \frac{S_{2} \Omega}{\left(k^{2}+4 \Omega^{2}\right)^{3 / 2}} \\
& +\frac{\pi \epsilon^{2}}{2}\left(k L^{2} N_{1}+\frac{N_{2}}{k L^{2}}+\frac{N_{3}}{\left(k L^{2}\right)^{3}}\right) .
\end{aligned}
$$


The expectation value $\left\langle T_{00}(\omega, \boldsymbol{x})\right\rangle$ in position space $\boldsymbol{x}$ is obtained by the Fourier transformation,

$$
\left\langle T_{00}(\omega, \boldsymbol{x})\right\rangle=\int_{-\infty}^{\infty} \frac{d^{3} k}{(2 \pi)^{3}} Q(\omega, \boldsymbol{k}) e^{i \boldsymbol{k} \cdot \boldsymbol{x}} .
$$

So, in the far asymptotic region $|\boldsymbol{x}| \gg 1$, the integration is dominant for $k \rightarrow 0$. In this limit,

$$
\begin{aligned}
\lim _{k \rightarrow 0} Q_{0} & \simeq \frac{\pi \epsilon^{2}}{4 L^{2} \Omega}\left(C_{2}+\frac{3 C_{3} k^{2}}{16 L^{4} \Omega^{4}}-\frac{C_{3}}{2 L^{4} \Omega^{2}}\right) \\
& -\frac{\pi \epsilon^{2}}{2} S_{1} \ln k+\frac{\pi \epsilon^{2}}{8 L^{4} \Omega^{2}} S_{2} \\
& +\frac{\pi \epsilon^{2}}{2}\left(k L^{2} N_{1}+\frac{N_{2}}{k L^{2}}+\frac{N_{3}}{\left(k L^{2}\right)^{3}}\right) .
\end{aligned}
$$

The integral can be easily performed by differentiating appropriately modified versions of the standard formulas

$$
\begin{array}{lll}
\int \frac{d^{3} k}{(2 \pi)^{3}} \frac{e^{i \boldsymbol{k} \cdot \boldsymbol{x}}}{|\boldsymbol{k}|}=\frac{1}{2 \pi^{2} R^{2}}, & \int \frac{d^{3} k}{(2 \pi)^{3}} \frac{e^{i \boldsymbol{k} \cdot \boldsymbol{x}}}{|\boldsymbol{k}|^{2}}=\frac{1}{4 \pi R}, \\
\int \frac{d^{3} k}{(2 \pi)^{3}} \frac{e^{i \boldsymbol{k} \cdot \boldsymbol{x}}}{|\boldsymbol{k}|^{3}}=-\frac{\ln R}{2 \pi^{2}}, & \int \frac{d^{3} k}{(2 \pi)^{3}} \frac{e^{i \boldsymbol{k} \cdot \boldsymbol{x}}}{|\boldsymbol{k}|^{4}}=-\frac{\pi R}{8 \pi},
\end{array}
$$

where $R:=|\boldsymbol{x}|$. So, Fourier transform of every term except last term in Eq. (III.16) decays faster than $1 / R^{2} \quad 2$. The Fourier transform of the last term becomes

$$
\left\langle T_{00}(\omega, \boldsymbol{x})\right\rangle \sim \frac{\epsilon^{2} L^{2} \Omega^{4}}{96 \pi^{2} \alpha^{\prime} R^{2}} \delta(\omega) \sim \frac{\epsilon^{2} \Omega^{4} \sqrt{g_{\mathrm{YM}}^{2} N}}{96 \pi^{2} R^{2}} \delta(\omega) .
$$

Substituting this into Eq.(III.7), we obtain

$$
\int_{-\infty}^{\infty} d t\left\langle T_{00}(t, \boldsymbol{x})\right\rangle \sim \frac{\epsilon^{2} \Omega^{4} \sqrt{g_{\mathrm{YM}}^{2} N}}{96 \pi^{2} R^{2}}(2 \pi \delta(0))=\frac{\epsilon^{2} \Omega^{4} \sqrt{g_{\mathrm{YM}}^{2} N}}{96 \pi^{2} R^{2}} \int_{-\infty}^{\infty} d t
$$

This relation implies that the coefficient of $\delta(\omega)$ in Eq.(III.18) corresponds to the time averaged energy density of the radiation. The energy density $\left\langle T_{00}\right\rangle$ can be expressed by the acceleration, $\dot{v} \sim \epsilon \Omega^{2}$, as $\sqrt{g_{\mathrm{YM}}^{2} N} \dot{v}^{2} / R^{2}$.

Let us compare Eq. (III.18) with the times averaged energy density of electromagnetic radiation sourced by an oscillating electron along $x_{1}$ axis with the same amplitude $\epsilon$ and frequency $\Omega$. In terms of charge $e$ of the electron, the power radiated per unit solid angle becomes

$$
\frac{e^{2} \epsilon^{2} \Omega^{4}}{32 \pi^{2} R^{2}} \sin ^{2} \Theta \propto \frac{e^{2} \dot{v}^{2}}{R^{2}} \sin ^{2} \Theta
$$

where $\Theta$ is the angle between $x_{1}$ axis and $\boldsymbol{x}$. Then the time averaged energy density is obtained by Eq.(III.20) divided by the speed of light.

So, both of strongly coupled gauge field and electromagnetic radiation densities agree on the asymptotic falloff and the acceleration dependence, while the angular distribution and the coupling dependence in the energy density are quite different. As seen in the case of a static quark [23], the

\footnotetext{
${ }^{2} \ln k$ term in Eq. (III.16) also decays faster than $1 / R^{2}$.
} 
energy density in Eq. (III.18) is proportional to the square of 't Hooft coupling, $\sqrt{g_{\mathrm{YM}}^{2} N}$, which is characteristic of strong coupling. We will argue on the angular distribution in Sec V

To investigate the energy loss rate of the accelerated quark, we need to obtain Poynting vector $\left\langle T^{0 i}\right\rangle$. Unfortunately, it is technically difficult to explicitly calculate $\left\langle T^{0 i}\right\rangle$ because of the complicated source (II.14) for the oscillating string solution (III.3). So, in the next section, we simply estimate the Poynting vector $\left\langle T^{0 i}\right\rangle$ by using a toy model where a straight string which moves at constant velocity and then suddenly stops.

\section{BREMSSTRAHLUNG RADIATION FROM A SCATTERED QUARK}

In this section, we shall consider bremsstrahlung radiation from a string which moves at constant velocity $v$ along $x_{1}$-axis, suddenly stops at the origin and then remains at rest. The Fourier transform $j(t, \boldsymbol{k}, r)$ of the source $j(\omega, \boldsymbol{k}, r)$ in Eq. (II.13) can be constructed by the source term $J(t, \boldsymbol{k}, r ; v)$ of the string moving at constant velocity $v$ for $-\infty<t<\infty$ as

$$
j(t, \boldsymbol{k}, r)=J(t, \boldsymbol{k}, r ; v) \theta(-t)+J(t, \boldsymbol{k}, r ; 0) \theta(t),
$$

where the time when the string stops is set to 0 .

So, using Fourier transform $j(\omega, \boldsymbol{k}, r)$ of $j(t, \boldsymbol{k}, r)$, we rewrite the master equation in Eq. (II.13) as

$$
\mathcal{L} \Phi=-j(\omega, \boldsymbol{k}, r)=-i\left[\frac{J(\boldsymbol{k}, r ; v)}{\omega_{-}-k_{1} v}-\frac{J(\boldsymbol{k}, r ; 0)}{\omega_{+}}\right], \quad \omega_{ \pm}=\omega \pm i \epsilon,
$$

where the source term of the string moving at constant velocity $v$ is given by [19]

$$
\begin{aligned}
J(\boldsymbol{k}, r ; v) & =\frac{l(v)}{6 k^{2}}\left\{k^{2}\left(2+v^{2}\right)-3 v^{2} k_{1}^{2}\right\}\left(-\frac{3}{r^{3}}+\frac{L^{4}}{r^{5}}\left(2 k^{2}-3 v^{2} k_{1}^{2}\right)\right), \\
l(v) & =\frac{1}{2 \pi \alpha^{\prime}} \frac{1}{\sqrt{1-v^{2}}} .
\end{aligned}
$$

In order to construct two independent solutions $\Phi_{H}, \Phi_{B}$ which satisfy the homogeneous equation $\mathcal{L} \Phi=0$ as well as the boundary conditions at the horizon and infinity, we introduce "radial wave number" in the bulk, $q:=\left(\omega^{2}-k^{2}\right)^{1 / 2}$. For the multivalued function $q$ in the complex $\omega$-plane, we take the branch cut such that $q$ is holomorphic in the upper plane, that is,

$$
q= \begin{cases}\sqrt{\omega^{2}-k^{2}} & \text { for } \omega>k \\ i \sqrt{k^{2}-\omega^{2}} & \text { for } k>\omega>-k . \\ -\sqrt{\omega^{2}-k^{2}} & \text { for }-k>\omega\end{cases}
$$

Then $\Phi_{H}$ and $\Phi_{B}$ are given by the Bessel functions as

$$
\Phi_{B}(r)=\frac{1}{r^{2}} J_{0}\left(q L^{2} / r\right), \quad \Phi_{H}(r)=\frac{1}{r^{2}} H_{0}^{(1)}\left(q L^{2} / r\right), \quad W=\frac{2 i}{\pi r^{5}} .
$$

Following to the procedure in the previous section, we obtain the coefficient $Q$ in Eq. (II.20) as

$$
Q=-\frac{L^{2}}{8 \alpha^{\prime} k^{2}}\left[\frac{\left\{3 v^{2} k_{1}^{2}-k^{2}\left(2+v^{2}\right)\right\}\left(k^{2}+3 v^{2} k_{1}^{2}-3 \omega^{2}\right)}{3 q\left(\omega_{-}-k_{1} v\right) \sqrt{1-v^{2}}}+\frac{2 k^{2}\left(k^{2}-3 \omega^{2}\right)}{3 \omega_{+} q}\right] .
$$

Supposing that $|v| \ll 1$, we shall expand $Q$ in terms of $v$ as

$$
\begin{aligned}
Q & =(\text { terms up to } v) \\
& +v^{2} \frac{L^{2}}{\alpha^{\prime}} \frac{2 k^{2} k_{1}^{2}\left(k^{2}-3 \omega^{2}\right)+\left\{2 k^{4}+9 k_{1}^{2} \omega^{2}+3 k^{2}\left(k_{1}^{2}-2 \omega^{2}\right)\right\} \omega_{-}^{2}}{24 k^{2} \omega_{-}^{3} q}+O\left(v^{3}\right) .
\end{aligned}
$$


The expansion would be valid for $|\omega|>\left|k_{1} v\right|$. So, hereafter, we focus on the high frequency behavior of the Poynting vector and we simply replace $\omega_{-}$with $\omega$.

Since the Poynting vector $\left\langle T^{0 i}(\omega, \boldsymbol{x})\right\rangle$ is defined by [19] as

$$
\left\langle T^{0 i}(\omega, \boldsymbol{x})\right\rangle=\int \frac{d^{3} k}{(2 \pi)^{3}} \frac{k_{i} \omega}{k^{2}} Q e^{i \boldsymbol{k} \cdot \boldsymbol{x}}=-i \frac{\partial}{\partial x^{i}}\left[\int \frac{d^{3} k}{(2 \pi)^{3}} \frac{\omega}{k^{2}} Q e^{i \boldsymbol{k} \cdot \boldsymbol{x}}\right],
$$

the total instantaneous power radiated by the quark is given by

$$
P(t)=\oint_{\partial V}\left\langle T^{0 i}(t, \boldsymbol{x})\right\rangle n_{i},
$$

where $n_{i}$ is a unit normal vector to two-dimensional spacelike surface $\partial V$ enclosing the quark. The first order terms of $v$ in Eq. (IV.7) does not contribute to the total instantaneous power $P$, since $\left\langle T^{0 i}\right\rangle n_{i} \leftrightarrow-\left\langle T^{0 i}\right\rangle n_{i}$ for $x_{1} \leftrightarrow-x_{1}$ for the first order terms. Therefore, we only calculate the second order terms of $v$.

After a little bit tedious calculation (in detail, see Appendix B), one gets the Poynting vector $\left\langle T^{0 i}\right\rangle$ as

$$
\begin{aligned}
\left\langle T^{0 i}(\omega, \boldsymbol{x})\right\rangle= & \text { (terms up to } v) \\
& -\frac{i L^{2} v^{2}}{4 \alpha^{\prime}} \frac{\partial}{\partial x^{i}}\left(\frac{I_{0}(R)}{3}-\frac{1}{3 \omega^{2}} \frac{\partial^{2}}{\partial x_{1}^{2}} I_{0}(R)+\frac{1}{2} \frac{\partial^{2}}{\partial x_{1}^{2}} I_{2}(R)-\omega^{2} I_{2}(R)-\frac{3 \omega^{2}}{2} \frac{\partial^{2}}{\partial x_{1}^{2}} I_{4}(R)\right) \\
& +O\left(v^{3}\right),
\end{aligned}
$$

where $R=|\boldsymbol{x}|$ and $I_{0}, I_{1}, I_{2}$, and $I_{4}$ are functions of $R$ and $\omega$ defined by

$$
\begin{aligned}
& I_{0}(R)=\frac{\omega}{4 \pi R} \times\left\{\begin{array}{ll}
H_{1}^{(1)}(|\omega| R) & \text { for } \omega>0 \\
H_{1}^{(2)}(|\omega| R) & \text { for } \omega<0
\end{array},\right. \\
& I_{1}(R)=\frac{i}{4 \pi R} \times\left\{\begin{array}{ll}
-H_{0}^{(1)}(|\omega| R) & \text { for } \omega>0 \\
H_{0}^{(2)}(|\omega| R) & \text { for } \omega<0
\end{array},\right. \\
& I_{2}(R)=\frac{1}{4 \pi R|\omega|} \int_{0}^{|\omega| R} d z \times\left\{\begin{array}{ll}
H_{0}^{(1)}(z) & \text { for } \omega>0 \\
\left(-H_{0}^{(2)}(z)\right) & \text { for } \omega<0
\end{array},\right. \\
& I_{4}(R)=-\frac{1}{R}\left[\int^{R}\left(\int^{\hat{r}} \tilde{r} I_{2}(\tilde{r}) d \tilde{r}\right) d \hat{r}+C_{1}(\omega) R+C_{2}(\omega)\right] \text {, }
\end{aligned}
$$

with two arbitrary functions $C_{1}$ and $C_{2}$ of $\omega$.

In the asymptotic region $R \rightarrow \infty$, the Hankel functions $H_{0}^{(n)}(|\omega| R)$ behaves as

$$
\begin{aligned}
H_{0}^{(1)}(|\omega| R) & \simeq \sqrt{\frac{2}{\pi}} \frac{e^{i|\omega| R}}{\sqrt{|\omega| R}}, & H_{0}^{(2)}(|\omega| R) & \simeq \sqrt{\frac{2}{\pi}} \frac{e^{-i|\omega| R}}{\sqrt{|\omega| R}}, \\
H_{1}^{(1)}(|\omega| R) & \simeq-i \sqrt{\frac{2}{\pi}} \frac{e^{i|\omega| R}}{\sqrt{|\omega| R}}, & H_{1}^{(2)}(|\omega| R) & \simeq i \sqrt{\frac{2}{\pi}} \frac{e^{-i|\omega| R}}{\sqrt{|\omega| R}} .
\end{aligned}
$$

So, in the frequency $\omega$-space, the leading order of $\left\langle T^{0 i}(\omega, \boldsymbol{x})\right\rangle$ becomes

$$
\left\langle T^{0 i}(\omega, R)\right\rangle \simeq-\frac{i L^{2} v^{2} \omega x^{i}}{12 \pi \alpha^{\prime} R^{2}}\left(\frac{2 x_{1}^{2}}{R^{2}}-1\right) \sqrt{\frac{|\omega|}{2 \pi R}} e^{i \omega R}=O\left(\frac{|\omega|^{3 / 2}}{R^{3 / 2}}\right) .
$$


The factor $e^{i \omega R}$ shows that the energy flux is an outgoing wave, as we expect. Since this factor oscillates heavily with respect to $\omega$ for large $R$, we might expect that the slow falloff with $R$ in Eq. (IV.13) disappears in position space except the neighborhood of future light cone $t \sim R$. In fact, the first term in Eq. (IV.10), for example, becomes in position space

$$
\left|\int_{-\infty}^{\infty} d \omega e^{-i \omega t} \frac{\partial I_{0}(R)}{\partial x^{i}}\right| \sim \frac{\left|x^{i}\right| \theta(t-R)}{\left(t^{2}-R^{2}\right)^{5 / 2}} \leq \frac{R \theta(t-R)}{\left(t^{2}-R^{2}\right)^{5 / 2}} \leq \frac{\theta(t-R)}{t^{4}\left(1-(R / t)^{2}\right)^{5 / 2}},
$$

where the step function inevitably appears from the causality condition. It is easily seen that in any timelike directions $(t>R)$ the term rapidly decays as $t^{-4}$. One can also see that the other terms in Eq. (IV.10) decays similarly. So, the slow falloff with $R$ in Eq. (IV.13) disappears in the real space. ${ }^{3}$ The strong divergence on the light cone $t \sim R$ may be due to the simple model in which the string suddenly stops.

In terms of 't Hooft coupling $g_{\mathrm{YM}}^{2} N$, Fourier transform of the total instantaneous power $P(\omega)$ is obtained from Eq.(IV.13) as

$$
P(\omega)=\oint_{\partial V}\left\langle T^{0 i}(\omega, R)\right\rangle n_{i} \simeq \frac{i v^{2} \omega}{9} \sqrt{|\omega| \frac{g_{\mathrm{YM}}^{2} N}{2 \pi}} R^{1 / 2} e^{i \omega R} .
$$

It is interesting to compare Eq. (IV.14 with the known result in the case of electromagnetism. In the electromagnetic case, we consider the bremsstrahlung radiation sourced by an electron with initial velocity $v$ which rapidly stops in the interval $\Delta t$. In the low velocity limit $v \rightarrow 0$, Fourier transform of the total instantaneous power radiated is given by

$$
P(\omega)=\oint\left\langle T^{0 i}(\omega, r)\right\rangle n_{i} \simeq-\frac{2 e^{2} v^{2}}{3 \Delta t} e^{i \omega R} .
$$

Eq. (IV.14) does not include the time interval $\Delta t$ since the quark suddenly stops with almost zero time interval. So, we must compare Eq. (IV.14) with Eq. (IV.15) in the range $\left|k_{1} v\right| \ll|\omega| \ll 1 / \Delta t$. We find that both the coupling dependence and the frequency dependence are quite different between the strongly coupled gauge theories and electromagnetism.

\section{CONCLUSION AND DISCUSSIONS}

We have examined radiation by an accelerated quark in $\mathcal{N}=4 \mathrm{SYM}$ theory with large 't Hooft coupling via gauge/gravity duality. In the oscillating quark with frequency $\Omega$, we have shown that the time averaged energy density far away from the quark correctly falls off as $\Omega^{4} / R^{2}$. The $\Omega$ dependence also agrees with the result in electromagnetism.

Interestingly, the angular distribution of the time averaged energy density is isotropic. Since $\mathcal{N}=4$ SYM theory is in deconfined phase, gluons can be emitted. Although the energy density is composed of not only gluons, but also other fields, it is natural to consider that the dominant ingredient of the energy density is gluon. In the weak coupling limit, the gluon radiation should become dipole radiation analogously with electromagnetism. So, one may expect that dipole radiation occurs even in $\mathcal{N}=4$ SYM theory. In the strongly coupled gauge theory, however, a gluon emitted by the source quark induces production of gluons around it by the self-interaction. As a result, we may expect that the energy density is asymptotically averaged over all the angles by the gluons.

\footnotetext{
${ }^{3}$ Since the expansion of Eq. (IV.6) by $v$ breaks down near the zero mode, we cannot strictly derive the energy flux in position space. From the dimensional analysis, however, we expect the power law decay in time.
} 
In the toy model of a scattered quark, we derived the total instantaneous power sourced by a quark which moves at constant velocity $v$, suddenly stops at the origin and then remains at rest by calculating Poynting vector $\left\langle T^{0 i}\right\rangle$ via gauge/gravity duality. In this case, we found that both the coupling dependence and the frequency dependence are quite different from the case of electromagnetism. The Fourier transform of the total instantaneous power in electromagnetism $P(\omega)$ is independent of the frequency $\omega$, while $P(\omega)$ in $\mathcal{N}=4$ SYM increases with $\omega$.

Even though the static force in strongly coupled gauge theory is well understood in terms of Wilson line probe, the radiation problem is still largely open question. In this paper we have investigated properties of radiation at zero temperature as a first step toward the understanding of the properties at finite temperature. It is still an open question whether or not universality exists in the radiation properties, although we have seen it in the ratio of viscosity divided by entropy density. If universality exists, we expect that the investigation at finite temperature may be connected to the RHIC data through jet quenching phenomenon. Even though there is no universality in the properties of radiation, we would obtain some insight into the understanding of the mechanism of energy dissipation by an external quark moving through the thermal plasma.

\section{Acknowledgments}

We would like to thank M. Natsuume for useful discussions. We are grateful to K. Matsui for comments.

\section{APPENDIX A: CALCULATION OF COEFFICIENT $Q$ FOR OSCILLATING STRING}

Since the coefficient $Q$ in Eq.(II.20) is finite part of $B$ in Eq.(II.19), we give calculation of $B$ in this appendix.

Let us define $j_{0 C}, j_{0 S}$, and $j_{0 N}$ as

$$
\begin{aligned}
& j_{0 C}:=\epsilon^{2}\left(\frac{C_{1}}{r^{3}}+\frac{C_{2}}{r^{5}}+\frac{C_{3}}{r^{7}}\right) \cos (2 \Omega z), \\
& j_{0 S}:=\epsilon^{2}\left(\frac{S_{1}}{r^{4}}+\frac{S_{2}}{r^{6}}\right) \sin (2 \Omega z), \\
& j_{0 N}:=\epsilon^{2}\left(\frac{N_{1}}{r^{3}}+\frac{N_{2}}{r^{5}}+\frac{N_{3}}{r^{7}}\right) .
\end{aligned}
$$

The coefficient $B_{S}$ coming from the partial source $j_{0 S}$ can be calculated as

$$
\begin{aligned}
B_{S}(r)= & \epsilon^{2} \int_{0}^{r} d r_{0}\left(\frac{S_{1}}{r_{0}}+\frac{S_{2}}{r_{0}^{3}}\right) K_{0}\left(k L^{2} / r_{0}\right) \sin \left(2 \Omega L^{2} / r_{0}\right) \\
= & S_{1} \epsilon^{2} \sqrt{\pi L^{2} \Omega} \int_{1 / r}^{\infty} d z_{0} z_{0}^{-1 / 2} K_{0}\left(k L^{2} z_{0}\right) J_{1 / 2}\left(2 L^{2} \Omega z_{0}\right) \\
& +S_{2} \epsilon^{2} \sqrt{\pi L^{2} \Omega} \int_{1 / r}^{\infty} d z_{0} z_{0}^{3 / 2} K_{0}\left(k L^{2} z_{0}\right) J_{1 / 2}\left(2 L^{2} \Omega z_{0}\right) .
\end{aligned}
$$

So, in the large $r$ limit, we obtain the coefficient $B_{S}$ as

$$
B_{S}=\frac{\pi}{2} S_{1} \epsilon^{2} \ln \left(\frac{2 \Omega}{k}+\sqrt{\frac{4 \Omega^{2}}{k^{2}}+1}\right)+\frac{\pi \Omega S_{2} \epsilon^{2}}{L^{4}\left(k^{2}+4 \Omega^{2}\right)^{3 / 2}}
$$


with the help of Appendix C.

The coefficient $B_{N}$ coming from the partial source $j_{0 N}$ can be calculated as

$$
\begin{aligned}
B_{N}(r)= & \epsilon^{2} \int_{0}^{r} d r_{0}\left(N_{1}+\frac{N_{2}}{r_{0}^{2}}+\frac{N_{3}}{r_{0}^{4}}\right) K_{0}\left(k L^{2} / r_{0}\right) \\
= & \epsilon^{2} \int_{k L^{2} / r}^{\infty} d y\left(\frac{k L^{2} N_{1}}{y^{2}}+\frac{N_{2}}{k L^{2}}+\frac{N_{3} y^{2}}{\left(k L^{2}\right)^{3}}\right) K_{0}(y) \\
= & -\epsilon^{2} N_{1} k L^{2}\left(\left[\frac{K_{0}(y)}{y}\right]_{k L^{2} / r}^{\infty}+\int_{k L^{2} / r}^{\infty} d y \frac{K_{1}(y)}{y}\right) \\
& +\frac{\epsilon^{2} N_{2}}{k L^{2}} \int_{k L^{2} / r}^{\infty} d y K_{0}(y)+\frac{\epsilon^{2} N_{3}}{\left(k L^{2}\right)^{3}} \int_{k L^{2} / r}^{\infty} d y y^{2} K_{0}(y) \\
= & -\epsilon^{2} N_{1} k L^{2}\left(\left[\frac{K_{0}(y)}{y}\right]_{k L^{2} / r}^{\infty}-\left[K_{1}(y)\right]_{k L^{2} / r}^{\infty}\right) \\
& +\epsilon^{2}\left(N_{1} k L^{2}+\frac{N_{2}}{k L^{2}}\right) \int_{k L^{2} / r}^{\infty} d y K_{0}(y)+\frac{\epsilon^{2} N_{3}}{\left(k L^{2}\right)^{3}} \int_{k L^{2} / r}^{\infty} d y y^{2} K_{0}(y),
\end{aligned}
$$

where we used the formulas

$$
K_{0}(y)-K_{2}(y)=-\frac{2}{y} K_{1}(y), \quad K_{0}(y)+K_{2}(y)=-2 K_{1}^{\prime}(y),
$$

in the third and fourth lines, respectively. In the large $r$ limit $(r \rightarrow \infty)$, we obtain the coefficient $B_{N}$ as

$$
B_{N}=\frac{\pi}{2} \epsilon^{2}\left(k L^{2} N_{1}+\frac{N_{2}}{k L^{2}}+\frac{N_{3}}{\left(k L^{2}\right)^{3}}\right)+O(r \ln r),
$$

with the help of formulas $\int_{0}^{\infty} K_{0}(y) d y=\int_{0}^{\infty} y^{2} K_{0}(y) d y=\pi / 2$.

The coefficient $B_{C}$ coming from the partial source $j_{0 C}$ can be calculated in the large $r$ limit as

$$
\begin{aligned}
B_{C}(r) & =\epsilon^{2} \int_{0}^{r} d r_{0}\left(C_{1}+\frac{C_{2}}{r_{0}^{2}}+\frac{C_{3}}{r_{0}^{4}}\right) K_{0}\left(k L^{2} / r_{0}\right) \cos \left(2 L^{2} \Omega / r_{0}\right) \\
& =\frac{\epsilon^{2} k L}{2} \sqrt{\frac{\pi}{\Omega}} \int_{1 / r}^{\infty} d z_{0}\left(C_{2} z_{0}^{1 / 2}+C_{3} z_{0}^{5 / 2}\right) K_{1}\left(k L^{2} z_{0}\right) J_{1 / 2}\left(2 L^{2} \Omega z_{0}\right) \\
& -\frac{\epsilon^{2} C_{3}}{L} \sqrt{\frac{\pi}{\Omega}} \int_{1 / r}^{\infty} d z_{0} z_{0}^{3 / 2} K_{0}\left(k L^{2} z_{0}\right) J_{1 / 2}\left(2 L^{2} \Omega z_{0}\right)+\epsilon^{2} C_{1} L^{2} \int_{L^{2} / r}^{\infty} d z_{0} \frac{K_{0}\left(k z_{0}\right)}{z_{0}^{2}} \cos \left(2 \Omega z_{0}\right) \\
& \simeq \frac{\pi \epsilon^{2} C_{2}}{2 L^{2} \sqrt{k^{2}+4 \Omega^{2}}}+\frac{\pi \epsilon^{2} C_{3}\left(k^{2}-8 \Omega^{2}\right)}{2 L^{6}\left(k^{2}+4 \Omega^{2}\right)^{5 / 2}}+\epsilon^{2} C_{1} L^{2} \int_{L^{2} / r}^{\infty} d z_{0} \frac{K_{0}\left(k z_{0}\right)}{z_{0}^{2}} \cos \left(2 \Omega z_{0}\right)
\end{aligned}
$$

with the help of Appendix C. The third term in the last line includes divergent terms. So, using 
Eq. (C.3), we carefully calculate as

$$
\begin{aligned}
P(r) & :=\epsilon^{2} C_{1} L^{2} \int_{L^{2} / r}^{\infty} d z_{0} \frac{K_{0}\left(k z_{0}\right)}{z_{0}^{2}} \cos \left(2 \Omega z_{0}\right) \\
& =\epsilon^{2} C_{1} r \cos b \int_{0}^{\infty} d t \frac{\cos (a t)}{\sqrt{t^{2}+1}}+\frac{\epsilon^{2} C_{1} r}{2} \int_{0}^{\infty} \frac{d t}{\sqrt{t^{2}+1}}\{(a t+b) \operatorname{si}(a t+b)+(a t-b) \operatorname{si}(a t-b)\} \\
& \simeq \epsilon^{2} C_{1} r \cos b K_{0}(a)-\epsilon^{2} C_{1} r \int_{0}^{\infty} \frac{d t}{\sqrt{t^{2}+1}}\left\{a t \operatorname{si}(a t)-\frac{b^{2}}{2} \cos (a t)-\frac{b^{2}}{2 t} \sin (a t)\right\} \\
& =\epsilon^{2} C_{1} r\left(K_{0}(a) \cos b+a \int_{a}^{\infty} d x \frac{K_{1}(x)}{x}+\frac{b^{2}}{2} K_{0}(a)+\frac{b^{2}}{2 a} \int_{0}^{a} d x K_{0}(x)\right)
\end{aligned}
$$

where $a=k L^{2} / r$ and $b=2 \Omega L^{2} / r$ and the function $\operatorname{si}(x)$ is defined by

$$
\operatorname{si}(x):=-\int_{x}^{\infty} d t \frac{\sin t}{t} .
$$

In the third line, we used the Taylor expansion of si $(x)$ around $x=a t$ for large $r$. In the asymptotic region, $r \rightarrow \infty$, we obtain

$$
P \sim O(r \ln r) .
$$

This logarithmic divergence does not appear in Eq. (II.20) because the coefficient $A$ in Eq. (II.19) also includes the same divergence with an opposite sign. So, $P$ does not contribute to $Q(\omega, \mathbf{k})$ in Eq. (II.20). 


\section{APPENDIX B: CALCULATION OF $\left\langle T^{0 i}\right\rangle$ FOR A SCATTERED STRING}

The calculation of $\left\langle T^{0 i}\right\rangle$ can be easily computed by differentiating appropriately modified versions of the following Fourier transforms of $1 / q$ and $1 / k q$ :

$$
\begin{aligned}
& I_{0}(R):=\frac{1}{(2 \pi)^{3}} \int d^{3} k \frac{e^{i \boldsymbol{k} \cdot \boldsymbol{x}}}{q} \\
& =\frac{1}{(2 \pi)^{2} i R} \int_{-\infty}^{\infty} d k \frac{k e^{i k R}}{q} \\
& =\frac{1}{(2 \pi)^{2} i R}\left[\int_{-|\omega|}^{|\omega|} d k \frac{k e^{i k R} \operatorname{sgn}(\omega)}{\sqrt{\omega^{2}-k^{2}}}+\int_{-\infty}^{\infty} d k \frac{\theta(|k|-|\omega|) k e^{i k R}}{i \sqrt{k^{2}-\omega^{2}}}\right] \\
& =\frac{1}{(2 \pi)^{2} i R}\left[\pi i \omega J_{1}(|\omega| R)-\frac{\partial}{\partial r} \int_{-\infty}^{\infty} d k \frac{\theta(|k|-|\omega|) e^{i k R}}{\sqrt{k^{2}-\omega^{2}}}\right] \\
& =\frac{1}{(2 \pi)^{2} i R}\left[\pi i \omega J_{1}(|\omega| R)+\pi \frac{\partial}{\partial R} N_{0}(|\omega| R)\right] \\
& =\frac{\omega}{4 \pi R} \times\left\{\begin{array}{ll}
H_{1}^{(1)}(|\omega| R) & \text { for } \omega>0 \\
H_{1}^{(2)}(|\omega| R) & \text { for } \omega<0
\end{array},\right. \\
& I_{1}(R):=\frac{1}{(2 \pi)^{3}} \int d^{3} k \frac{e^{i \boldsymbol{k} \cdot \boldsymbol{x}}}{q k} \\
& =\frac{1}{(2 \pi)^{2} i R}\left[\int_{-|\omega|}^{|\omega|} d k \frac{e^{i k R} \operatorname{sgn}(\omega)}{\sqrt{\omega^{2}-k^{2}}}+\int_{-\infty}^{\infty} d k \frac{\theta(|k|-|\omega|) e^{i k R}}{i \sqrt{k^{2}-\omega^{2}}}\right] \\
& =\frac{1}{(2 \pi)^{2} i R}\left[\pi \operatorname{sgn}(\omega) J_{0}(|\omega| R)+\pi i N_{0}(|\omega| R)\right] \\
& =\frac{i}{4 \pi R} \times\left\{\begin{array}{ll}
-H_{0}^{(1)}(|\omega| R) & \text { for } \omega>0 \\
H_{0}^{(2)}(|\omega| R) & \text { for } \omega<0
\end{array}\right. \text {, }
\end{aligned}
$$

where $R=|\boldsymbol{x}|$. So, Fourier transformation of $1 / k^{2} q$ is easily calculated as

$$
\begin{aligned}
I_{2}(R) & :=\frac{1}{(2 \pi)^{3}} \int d^{3} k \frac{e^{i \boldsymbol{k} \cdot \boldsymbol{x}}}{k^{2} q} \\
& =\frac{1}{2 \pi^{2} R} \int_{0}^{\infty} d k \frac{\sin (k R)}{k q} \\
& =\frac{1}{2 \pi^{2} R} \int_{0}^{R} d \tilde{r}\left[\int_{0}^{\infty} d k \frac{\cos (k \tilde{r})}{q}\right] \\
& =\frac{i}{R} \int_{0}^{R} d \tilde{r} \tilde{r} I_{1}(\tilde{r})
\end{aligned}
$$

Similarly, from the fact that

$$
\frac{\partial^{2}}{\partial R^{2}}\left[\frac{R}{(2 \pi)^{3}} \int d^{3} k \frac{e^{i \boldsymbol{k} \cdot \boldsymbol{x}}}{k^{4} q}\right]=-R I_{2}(R),
$$


Fourier transformation of $1 / k^{4} q$ can be represented by two integration constants $C_{1}(\omega)$ and $C_{2}(\omega)$ as

$$
\begin{aligned}
I_{4}(R) & :=\frac{1}{(2 \pi)^{3}} \int d^{3} k \frac{e^{i \boldsymbol{k} \cdot \boldsymbol{x}}}{k^{4} q} \\
& =-\frac{1}{R}\left[\int^{R} d \hat{r}\left(\int^{\hat{r}} d \tilde{r} \tilde{r} I_{2}(\tilde{r})\right)+C_{1}(\omega) r+C_{2}(\omega)\right] .
\end{aligned}
$$

\section{APPENDIX C: FORMULAS}

For any real positive parameter $a$ and $b$, one obtains [24]

$$
\begin{aligned}
& \int_{0}^{\infty} d x x^{-1 / 2} K_{0}(a x) J_{1 / 2}(b x)=\sqrt{\frac{\pi}{2 b}} \sinh ^{-1}\left(\frac{b}{a}\right)=\sqrt{\frac{\pi}{2 b}} \ln \left(\frac{b}{a}+\sqrt{\frac{b^{2}}{a^{2}}+1}\right), \\
& \int_{0}^{\infty} d x x^{3 / 2} K_{0}(a x) J_{1 / 2}(b x)=\sqrt{\frac{\pi b}{2}} \frac{1}{\left(a^{2}+b^{2}\right)^{3 / 2}} .
\end{aligned}
$$

It is easy to get the following equations by differentiating Eq.(C.1) with respect to $a$,

$$
\begin{aligned}
& \int_{0}^{\infty} d x x^{1 / 2} K_{1}(a x) J_{1 / 2}(b x)=\sqrt{\frac{\pi b}{2}} \frac{1}{a\left(a^{2}+b^{2}\right)^{1 / 2}}, \\
& \int_{0}^{\infty} d x x^{5 / 2} K_{1}(a x) J_{1 / 2}(b x)=\sqrt{\frac{\pi b}{2}} \frac{3 a}{\left(a^{2}+b^{2}\right)^{5 / 2}} .
\end{aligned}
$$

In Eq.A.8, we use the integral representation of $K_{0}(x)$

$$
K_{0}(x)=\int_{0}^{\infty} d t \frac{\cos (x t)}{\sqrt{t^{2}+1}} .
$$

[1] "Why does the quark gluon plasma at RHIC behave as a nearly ideal fluid?," Prog. Part. Nucl. Phys. 53, 273 (2004) arXiv:hep-ph/0312227.

[2] "What RHIC experiments and theory tell us about properties of quark-gluon plasma?," Nucl. Phys. A750, 64 (2005) arXiv:hep-ph/0405066.

[3] J. Maldacena, "The Large N Limit of Superconformal Field Theories and Supergravity," Adv. Theor. Math. Phys. 2, 231 (1998) arXiv:hep-th/9711200.

[4] E. Witten, "Anti-de Sitter space and holography," Adv. Theor. Math. Phys. 2, 253 (1998) arXiv:hep-th/9802150.

[5] S.S. Gubser, I.R. Klebanov, and A.M. Polyakov, " Gauge Theory Correlators from Non-Critical String Theory," Phys. Lett. B428, 105 (1998) arXiv:hep-th/9802109.

[6] P. Kovtun, D. T. Son, and A. O. Starinets, "Viscosity in Strongly Interacting Quantum Field Theories from Black Hole Physics," Phys. Rev. Lett. 94, 111601 (2005) arXiv:hep-th/0405231.

[7] K. Maeda, M. Natsuume, and T. Okamura, "Viscosity of gauge theory plasma with a chemical potential from AdS/CFT correspondence," Phys. Rev. D73, 066013 (2006) arXiv:hep-th/0602010.

[8] C. P. Herzog, A. Karch, P. Kovtun, C. Kozcaz, and L. G. Yaffe, "Energy loss of a heavy quark moving through N=4 supersymmetric Yang-Mills plasma," J. High Energy Phys. 07 (2006) 013 arXiv:hep-th/0605158. 
[9] S. S. Gubser, "Drag force in AdS/CFT," Phys. Rev. D74, 126005 (2006) arXiv:hep-th/0605182 .

[10] C. P. Herzog, "Energy Loss of Heavy Quarks from Asymptotically AdS Geometries," J. High Energy Phys. 09 (2006) 032 arXiv:hep-th/0605191.

[11] H. Liu, K. Rajagopal, and U. A. Wiedemann, "Calculating the Jet Quenching Parameter from AdS/CFT," Phys. Rev. Lett. 97, 182301 (2006) arXiv:hep-th/0605178.

[12] J. Casalderrey-Solana and D. Teaney, "Heavy Quark Diffusion in Strongly Coupled $\mathcal{N}=4$ Yang Mills," Phys. Rev. D74, 085012 (2006) arXiv:hep-ph/0605199.

[13] A. Buchel, "On jet quenching parameters in strongly coupled non-conformal gauge theories," Phys. Rev. D74, 046006 (2006) arXiv:hep-th/0605178.

[14] E. Caceres and A. Guijosa, "Drag Force in a Charged N=4 SYM Plasma," J. High Energy Phys. 11 (2006) 077 arXiv:hep-th/0605235.

[15] J. J. Friess, S. S. Gubser, and G. Michalogiorgakis, "Dissipation from a heavy quark moving through N=4 super-Yang-Mills plasma," J. High Energy Phys. 09 (2006) 072 arXiv:hep-th/0605292 .

[16] S. S. Gubser and A. Yarom, "Universality of the diffusion wake in the gauge-string duality," Phys. Rev. D77, 066007 (2008) arXiv:0709.1089 [hep-th]] .

[17] S. S. Gubser, S. S. Pufu, and A. Yarom, "Sonic booms and diffusion wakes generated by a heavy quark in thermal AdS/CFT," Phys. Rev. Lett. 100, 012301 (2008) arXiv:0706.4307 [hep-th]] .

[18] S. S. Gubser, S. S. Pufu, and A. Yarom, "Energy disturbances due to a moving quark from gauge-string duality," J. High Energy Phys. 09 (2007) 108 [arXiv:0706.0213 [hep-th]] .

[19] S. S. Gubser and S. S. Pufu, "Master field treatment of metric perturbations sourced by the trailing string," Nucl. Phys. B790, 42 (2008) arXiv:hep-th/0703090.

[20] J. J. Friess, S. S. Gubser, G. Michalogiorgakis, and S. S. Pufu, "The stress tensor of a quark moving through N=4 thermal plasma," Phys. Rev. D75, 106003 (2007) arXiv:hep-th/0607022.

[21] C. G. Callan and A. Guijosa, "Undulating Strings and Gauge Theory Waves," Nucl. Phys. B565, 157 (2000) arXiv:hep-th/9906153.

[22] S.-J. Sin and I. Zahed, "Holography of Radiation and Jet Quenching," Phys. Lett. B608, 265 (2005) arXiv:hep-th/0407215.

[23] U. H. Danielsson, E. Keski-Vakkuri, and M. Kruczenski, "Vacua, Propagators, and Holographic Probes in AdS/CFT," J. High Energy Phys. 01 (1999) 002 arXiv:hep-th/9812007. .

[24] G.N. Watson, A Treatise on the Theory of Bessel Functions, 2nd ed., (Cambridge University Press, Cambridge, England, 1966) . 